\title{
Pulmonary Artery Hypertension as A Risk Factor for Long-Term Survival after Heart Transplantation
}

\author{
Milos Matkovic, MD, PhD, ${ }^{1,2}$ Vladimir Milicevic, MD, ${ }^{1}$ Ilija Bilbija, MD, ${ }^{1,2}$ Nemanja Aleksic, MD, ${ }^{1,2}$ \\ Marko Cubrilo, MD,${ }^{1}$ Emilija Nestorovic, MD, PhD,${ }^{1,2}$ Ana Antic, MD,${ }^{1}$ Mina Zlatkovic, MD, ${ }^{1}$ \\ Milos Velinovic, MD, PhD, ${ }^{1,2}$ Svetozar Putnik, $\mathrm{MD}, \mathrm{PhD}^{1,2}$ \\ ${ }^{1}$ Department for Cardiac Surgery, University Clinical Center of Serbia, Belgrade, Serbia; ${ }^{2}$ Faculty of Medicine, University of Belgrade, \\ Belgrade, Serbia
}

\section{ABSTRACT}

Background: Heart failure is the most frequent cause of pulmonary artery hypertension $(\mathrm{PAH})$ and its severity may predict the development of heart failure (HF) and is known to be a prognostic factor of poor outcome after heart transplant (HTx). The aim of this study was to investigate the impact of preoperative $\mathrm{PAH}$ related to left-sided HF on long-term survival after HTx and to identify the hemodynamic parameters of PAH that predict survival after HTx.

Methods: A prospective observational trial was performed, and it included 44 patients subjected to heart transplantation. Patients were divided into two groups: The first one with the preoperative diagnosis of $\mathrm{PAH}$ and the second one without the PAH diagnosed prior to the HTx. The two groups were compared for baseline characteristics, operative characteristics, survival, and hemodynamic parameters obtained by right heart catheterization. Survival was analyzed using Kaplan Meyer analysis, and Cox regression analysis was performed to determine independent predictors of survival.

Results: The median follow-up time was 637.4 days (1-2028 days). The median survival within the group of patients with preoperative PAH was 1144 days (95\% CI 662.884-1625.116) and 1918.920 days (95\% CI 1594.577-2243.263) within the group of patients without PAH $(P=.023)$, HR $0.279(95 \%$ [CI]: $0.086-0.910 ; P=.034$. The 30 -day mortality in patients within PAH group was significantly higher, six versus two patients in the non PAH group $\left(\chi^{2}=5.103, P<.05\right)$, while the long-term outcome after this period did not differ between the groups. Patients with preoperative PAH had significantly higher values of MPAP, PCWP, TPG and PVRI, while CO and CI did not differ between the two groups. Mean PVRI was $359.1 \pm 97.3 \mathrm{dyn} \cdot \mathrm{s} \cdot \mathrm{cm}^{-5}$ in the group with preoperative $\mathrm{PAH}$ and $232.2 \pm 22.75 \mathrm{dyn} \cdot \mathrm{s} \cdot \mathrm{cm}^{-5}$ in the group without PAH, $P<.001$. TPG values were $11.95 \pm 5.08 \mathrm{mmHg}$ in the PAH group while patients without $\mathrm{PAH}$ had mean values of $5.16 \pm$ $1.97 \mathrm{mmHg}, P<.001$. Cox regression analysis was done for

Received March 8, 2021; received in revised form March 21, 2021; accepted March 22, 2021.

Correspondence: Milos Matkovic, Department for Cardiac Surgery, Clinical Center of Serbia, 8 Koste Todorovica St., 11000, Belgrade, Serbia; +381113663201 (e-mail:dr:matko@hotmail.com). the aforementioned parameters. Hazard ratio for worse survival after HTx for elevated values of PVRI was $1.006(95 \%$ [CI]: 1.001-1.012; $P=.018)$ TPG had a hazard ratio of 1.172 (95\% [CI]: $1.032-1.233 ; P=.015$ ).

Conclusion: Pulmonary artery hypertension is an independent risk factor for higher 30-day mortality after HTx, while it does not affect the long-term outcome. Hemodynamic parameters obtained by right heart catheterization in heart transplant candidates could predict postoperative outcome. PVRI and TPG have been identified as independent predictors of higher 30-day postoperative mortality.

\section{INTRODUCTION}

Survival in patients with pulmonary artery hypertension $(\mathrm{PAH})$ related to the left heart failure without therapy is up to 1-2.8 years. Great efforts have been made in enhancing therapeutic options for this group of patients, as well in understanding the pathophysiological mechanisms [D'Alonzo 1991]. The exact pathophysiological mechanisms that lead to $\mathrm{PAH}$ in left heart failure (HF) still remain unclear, while the only therapy remains treatment of left-sided heart failure. Heart failure is the most frequent cause of $\mathrm{PAH}$ and its severity may predict the development of heart failure and is known to be a prognostic factor of poor outcome after heart transplant (HTx) [Mehra 2006].

Multiple trials have confirmed that elevated pulmonary vascular resistance (PVR) or transpulmonary gradient (TPG) is an independent risk factor for both early and late mortality after orthotopic HTx. This is mainly due to right ventricular failure because of the inability of the donor heart to meet the demands of elevated pulmonary pressure [Klotz 2006]. Also, the thresholds of hemodynamic parameters, such as PVR and TPG, that predict the onset of right heart failure and the outcome of heart transplant are not clearly established. The International Society for Heart and Lung Transplantation (ISHLT) suggests that fixed PAH with PVR $>2.5 \mathrm{WU}$ is a negative prognostic factor for survival after HTx, while PVR $>400$ dyn s cm${ }^{-5}$ ( > $5 \mathrm{WU}$ ) is considered to be a relative contraindication for surgery [Mehra 2016]. On the other hand, reversible PAH in patients, who are candidates for HTx, is considered to be a negative prognostic factor for long-term survival [Butler 2005]. 
Table 1. Preoperative patient characteristics

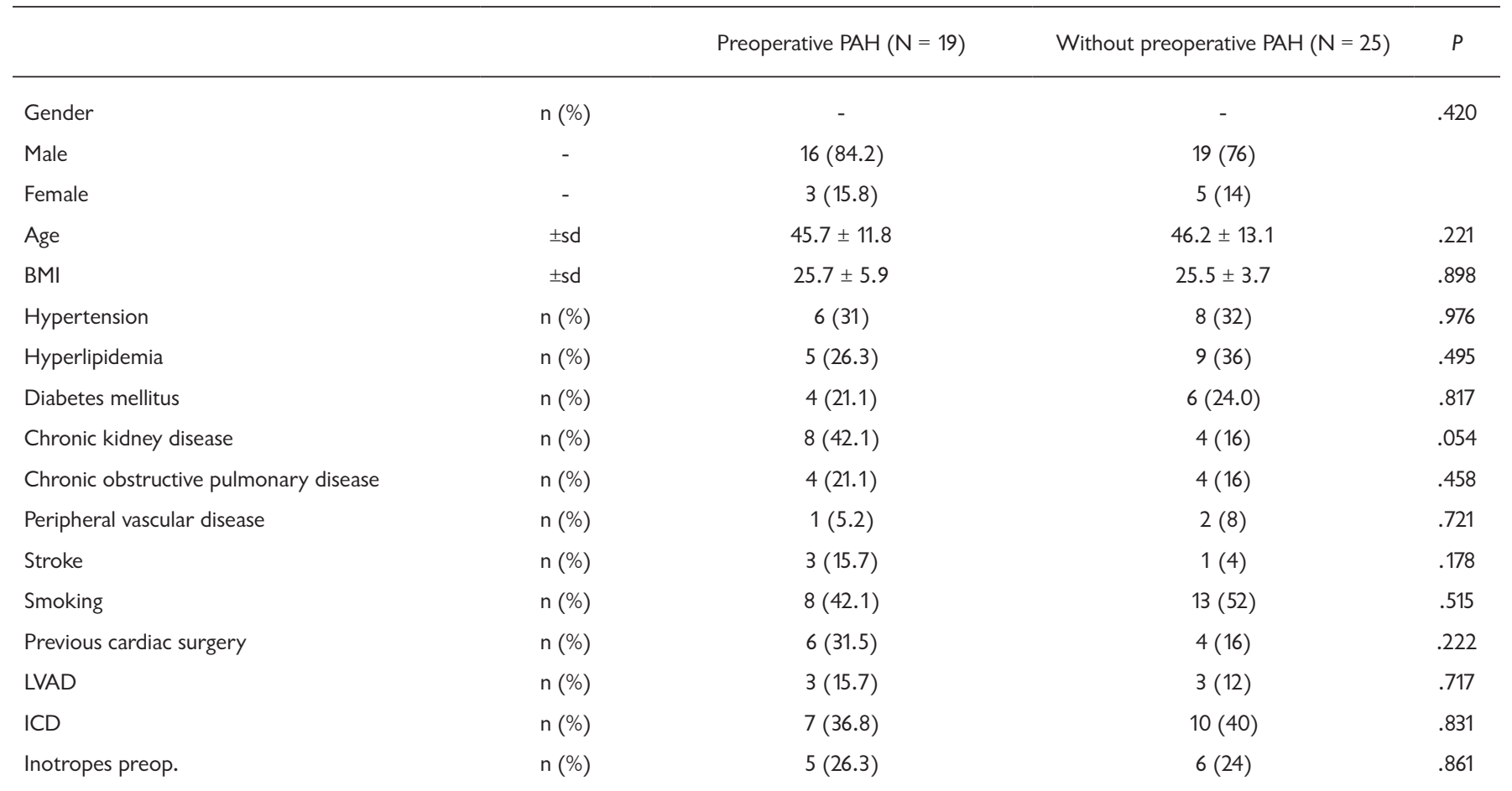

The aim of this study was to investigate the impact of preoperative PAH related to left-sided HF on long-term survival after HTx and to identify the hemodynamic parameters of PAH that predict survival after HTx.

\section{MATERIAL AND METHODS}

Study design and patient population: A prospective observational trial was performed. All patients who underwent heart transplant in our institution between September 2013 and February 2020 were enrolled in the trial - a total of 44 patients. The ethical committee of our institution waived the need for the informed consent, thus all patients had it signed before entering the trial. Further, patients were divided into two groups: the first one with the preoperative diagnosis of $\mathrm{PAH}$ and the second one without the PAH diagnosed prior to the HTx.

The diagnosis of PAH was established based on clinical criteria and the invasive hemodynamic measurements with Swan-Ganz right heart catheterization. Mean pulmonary artery pressure $>25 \mathrm{mmHg}$ was considered a threshold for the diagonosis of PAH. All patients had right heart catheterization done prior listing for $\mathrm{HTx}$, and it was repeated after six months if the patient sat longer on the waiting list, or if the left ventricular assist device (LVAD) was implanted. The listing criteria for HTx were according to present ISHLT guidelines. The last hemodynamic measurements before the HTx were taken for analysis.

Hemodynamic measurements and surgical technique: Transjugular approach under fluoroscopy guidance was used for Swan-Ganz catheterization (Baxter Health Care Corp, Santa Ana, CA, USA). Systolic and diastolic pulmonary artery pressure (SPAP and DPAP), mean pulmonary artery pressure (MPAP), pulmonary capillary wedge pressure (PCWP), mean pressure in right ventricle as well as mean and systolic arterial pressure were recorded. Continuous ECG monitoring was performed. Cardiac output (CO) was obtained with thermodilution method. Cardiac index (CI), stroke volume (SV), stroke volume index (SVI), PVR, PVR index, TPG, DPG were calculated with following formulas: $\mathrm{CI}=\mathrm{CO} / \mathrm{BSA}, \mathrm{SV}$ $=\mathrm{CO} / \mathrm{HR}, \mathrm{SVI}=\mathrm{SV} / \mathrm{BSA}, \mathrm{SVI}=0.0136, \mathrm{PVR}=\mathrm{TPG} / \mathrm{CO}$, PVRI $=$ TPG $/ C I$, TPG $=$ MPAP-PCWP, DPG $=$ DPAP $\mathrm{PCWP}$, and SVR $=(\mathrm{MAP}-\mathrm{MRAP}) / \mathrm{CO}$.

Surgery was done under general anesthesia with the use of bicaval cannulation technique for the establishment of cardiopulmonary bypass. Biatrial or bicaval technique was done according to the surgeon's preference. The donor heart was arrested with Custodiol solution and preserved on ice and in the Custodiol solution and transported, according to the local protocol. Nitric oxide systematically was administered to all patients with preoperative pulmonary hypertension.

Data collection: Patient characteristics, comorbidities, hemodynamic measurements, operative characteristics, as well as complications and intrahospital mortality were recorded from medical records. Mortality in the follow-up period was recorded from medical records, on scheduled follow-up exams for transplanted patients according to local protocol, and through telephone interview. The Euroscore, STS score, and ISHLT registry variable definitions were used.

Statystical analysis: Descriptive statistics were calculated for the baseline demographic and clinical features, as well as 


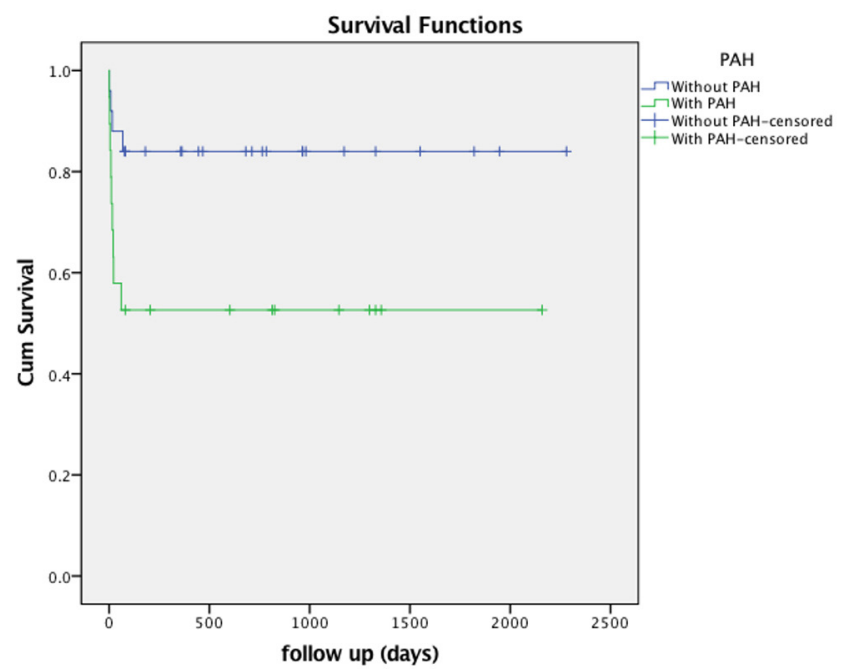

Figure 1. Kaplan-Meyer survival curves for the group with preoperative $\mathrm{PAH}$ and for the group without preoperative PAH.

treatment outcomes. Normality of distribution was tested by graphical and mathematical methods. Continuous variables were presented as mean $\pm \mathrm{SD}$. Categorical variables were presented as numbers and percentages. Differences between groups were analyzed using Student's t-test and MannWhitney test for continuous variables and the Pearson chisquared test for categorical variables. Survival was analyzed using Kaplan Meyer analysis and long-rank test was used to analyze differences between groups. Cox regression analysis was performed in order to determine independent predictors of survival. All analyses were performed with software SPSS (version 21.0 for Windows; SPSS; IBM, Chicago, IL, USA).

\section{RESULTS}

Preoperative baseline characteristics are shown in Table 1. The two groups did not differ significantly in gender-age distribution, comorbidities, previous cardiac surgery, or in the frequency of previously implanted LVAD or ICD.

Operative characteristics are shown in Table 2. The median age of our transplanted patient was 47 years, while the youngest was 16 years old and the oldest was 64 years. The two groups did not differ statistically in donor age, donor gender, ischemic time, surgical technique, or the number of surgical revisions.

The median follow-up time was 637.4 days (1-2028 days). The median survival within the group of patients with preoperative PAH was 1144 days (95\% CI 662.884-1625.116) and 1918.920 days (95\% CI 1594.577-2243.263) within the group of patients without PAH. The survival analysis showed statistically significant lower survival in the group of patients with preoperative PAH (log-rank $P=.023$ ) (Figure 1). The $\mathrm{HR}$ for the lower survival for patients with preoperative $\mathrm{PAH}$ was 0.279 (95\% [CI]: 0.086-0.910; $P=.034$ ) (Figure 2). After the subanalysis of 30 -day mortality, patients within the $\mathrm{PAH}$

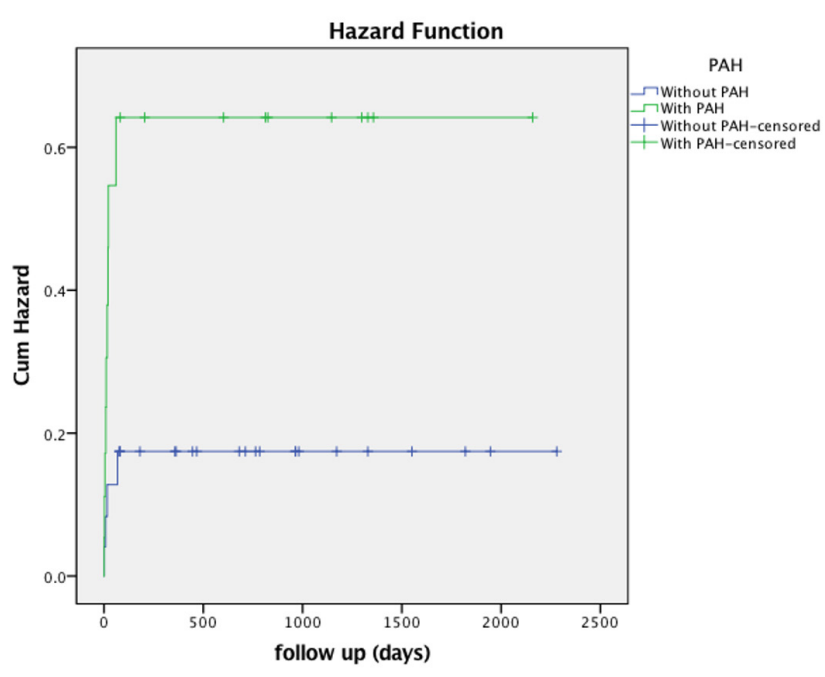

Figure 2. Hazard ratio for the group with preoperative $\mathrm{PAH}$ and for the group without preoperative PAH.

group had significantly higher mortality rate, six versus two patients in the non PAH group $\left(\chi^{2}=5.103, P<.05\right)$. However, the long-term mortality after the 30 -day postoperative period did not differ between the two groups $(P<.05)$. Mean ICU stay was significantly higher in the $\mathrm{PAH}$ group in comparison with the non PAH group $(12.3 \pm 7.4$ days versus $8.1 \pm 3.3$ days, $\left(\chi^{2}=3.707, P<.05\right)$, while mean intubation time did not differ between the two groups $(23.5 \pm 4.3$ hours versus $21.3 \pm 5.6$ hours, $\left(\chi^{2}=4.852, P>.05\right)$.

Preoperative MPAP, PCWP, PVRI, CO and CI were registered during hemodynamic measurements (Table 3). Patients with preoperative $\mathrm{PAH}$ had significantly higher values of MPAP, PCWP, TPG and PVRI, while CO and CI did not differ between the two groups. Mean PVRI was $359.1 \pm 97.3$ dyn $\cdot \mathrm{s} \cdot \mathrm{cm}^{-5}$ in the group with preoperative PAH and $232.2 \pm$ 22.75 dyn $\cdot \mathrm{s} \cdot \mathrm{cm}^{-5}$ in the group without PAH, $P<.001$. Preoperative TPG values were $11.95 \pm 5.08 \mathrm{mmHg}$ in the $\mathrm{PAH}$ group, while patients without $\mathrm{PAH}$ had mean values of 5.16 $\pm 1.97 \mathrm{mmHg}, P<.001$. Cox regression analysis was done for the aforementioned parameters. PVRI and TPG were shown to be statistically significant as independent predictors of lower survival after HTx. Preoperative MPAP, PCWP, CO, and CI were not statistically significant as independent predictors of survival for patients subjected to HTx. Hazard ratio for worse survival after HTx for elevated values of PVRI was $1.006(95 \%[\mathrm{CI}]: 1.001-1.012 ; P=.018)$. TPG had a hazard ratio of $1.172(95 \%[\mathrm{CI}]: 1.032-1.233 ; P=.015)$.

\section{DIscussion}

Despite all advances in the area of heart transplantation, right heart failure remains one of the most common causes of early mortality after HTx. The frequency of this complication goes up to $20 \%$ in the literature [Vassileva 2013]. In the event of recipient $\mathrm{PAH}$, the donor heart has not been 
Table 2. Operative characteristics

\begin{tabular}{|c|c|c|c|c|}
\hline Donor gender & $\mathrm{n}(\%)$ & - & - & .328 \\
\hline Female & - & $8(42.2)$ & $7(18)$ & \\
\hline Donor age & $\pm s d$ & $42.1 \pm 13.4$ years & $41.3 \pm 10.5$ years & .428 \\
\hline Ischemic time & $\pm s d$ & $126.1 \pm 41.4 \mathrm{mins}$ & $109.4 \pm 38.4 \mathrm{mins}$ & .705 \\
\hline Biatrial technique & $\mathrm{n}(\%)$ & $16(84.2)$ & $17(68)$ & .158 \\
\hline Surgical revision & $\mathrm{n}(\%)$ & $8(42.2)$ & $4(16)$ & .057 \\
\hline
\end{tabular}

conditioned for increased pulmonary resistance and has to use its reserve to cope with increased afterload. This setting can lead to acute or chronic right heart failure. Thus, the adequate hemodynamic diagnostic tools are necessary in order to identify patients who are at higher risk for development of right heart failure after HTx. Arrhythmias, including atrial fibrillation, have been identified as confounding risk factors alongside PAH for increased early mortality. Also, the physiological changes that occur within the donor heart caused by previous PAH and oxidative stress can trigger atrial fibrillation [Murali 1993]. The ISHLT guidelines suggest that SPAP $>60 \mathrm{mmHg}$ and $\mathrm{PVR}>5 \mathrm{WU}$ or PVRI > $6 \mathrm{WUm}^{-2}$ or TPG $>16-20 \mathrm{mmHg}$ present the high risk for postoperative right heart failure and early mortality, while PVR > 400 dyn s cm$~_{-5}$ (> $5 \mathrm{WU}$ ) is considered relative contraindication for HTx [Mehra 2006].

Rivinius et al performed a retrospective observational trial similar to our study and included 561 patients. The share of perioperative $\mathrm{PAH}$ was $16.8 \%$, while in our trial that number was $41 \%$. They have showed worse 30 -day mortality after HTx in patients with PAH, which was confirmed in our trial. Their multivariant analysis pointed out $\mathrm{PAH}$ as an independent negative predictor of early mortality with the HR: 4.4, 95\% CI: $2.5-7.6, P<.01$, also confirmed in our trial with HR: 0.279 (95\% [CI]: 0.086-0.910; $P=.034$ ) [Rivinius 2020]. After the subanalysis, after 30 days postoperatively, the long-term survival in our trial remained the same for the two groups.

Although the majority of large trials have identified PAH as a predictor of post-transplant mortality, there are several trials that have found that $\mathrm{PAH}$ is an independent predictor of poor outcome [Erickson 1990; Tenderich 1998; Lindelow 1999; Hosenpud 2000]. Murali et al identified PAH as a predictor of operative mortality, however this difference was not observed at 30-day mortality analysis [Murali 1993]. The possible reason for these results may be due to fact that all of these trials were experiences from a single center and included a small number of patients. Our trial, although a single center experience, has confirmed the results of larger trials stating that $\mathrm{PAH}$ is an independent risk factor. Also, these single center experiences did not take into consideration patients who were on LVAD as a bridge to transplant that can lower the $\mathrm{PAH}$ and influence the postoperative outcome. In our trial, $13.6 \%$ of patients had LVAD implanted preoperatively as a bridge to transplant.

A large ISHLT multicentric trial showed linear correlation between PVR values and one-year survival after HTx [Hosenpud 2000]. Vakil et al found that values of PVR $\geq$ 200 dyn.s.cm-5 carry increased risk for diminished 30-day survival [Vakil 2014]. It also has been established that mortality risk increases for every $80 \mathrm{dyn} \cdot \mathrm{s} \cdot \mathrm{cm}^{-5}$ of elevated PVR [Chang 2005]. Rabin et al demonstrated that even patients with mild elevateed PVR have increased mortality risk after HTx [Rabin 2019]. Mean PVR values in the group of patients with preoperative $\mathrm{PAH}$ and higher mortality in our trial was $359.1 \pm 97.3 \mathrm{dyn} \cdot \mathrm{s} \cdot \mathrm{cm}^{-5}$.

After investigating the predictive value of hemodymanic parameters of PAH, it has been established that TPG and PVRI are better predictors of worse outcome than PVR [Delgado 2001]. Also, as TPG is highly sensitive to changes in $\mathrm{CO}$ and left atrial pressure, diastolic pulmonary gradient has been shown to be an even better predictor, with values higher than $>7 \mathrm{mmHg}$ significantly predicting higher mortality after HTx [Costanzo 2010]. Trials that investigated the predictive value of these hemodynamic parameters were heterogenous in design and study populations, thus there is no consensus about the cut-off values that predict worse outcomes. Bourge et al performed one of the first trials that investigated prognostic value of hemoynamic parameters obtained by right heart catheterization and included 182 patients who had HTx performed [Bourge 1991]. They found the correlation between PVR, PVRI and TPG and elevated mortality one year after HTx. Also, Erickson et al confirmed the predictive value of PVRI and TPG on 109 transplanted patients. In their trial, patients with TPG $>12 \mathrm{mmHg}$ had five times higher risk of mortality after the surgery. PVRI was pointed out as an independent predictor of worse long-term outcome after HTx [Erickson 1990]. Moreira et al used the heart transplantation as a physiological model of hemodynamic changes in pulmonary vasculature and in the afterload. They have shown that PVR and TPG are independent predictors of worse long-term outcome. The decrease of PVR values after HTx was identified as positive predictor of better survival [Moreira 2015]. Rivinius et al showed that PVR $>300 \mathrm{dyn} \cdot \mathrm{s} \cdot \mathrm{cm}^{-5 a}$ and PVRI also are independent predictors of survival after HTx 
Table 3. Preoperative hemodynamic parameters for the group with preoperative PAH and for the group without preoperative $\mathrm{PAH}$

\begin{tabular}{lcccc}
\hline & & Preoperative PAH $(\mathrm{N}=19)$ & Without preoperative PAH $(\mathrm{N}=25)$ \\
\hline MPAP $(\mathrm{mmHg})$ & $\chi^{ \pm s d}$ & $35.4 \pm 9.15$ & $16.4 \pm 1.55$ & .001 \\
PCWP $(\mathrm{mmHg})$ & $\chi^{ \pm s d}$ & $22.53 \pm 4.28$ & $11.1 \pm 2.1$ & .027 \\
PVRI $\left(\mathrm{dyn} \cdot \mathrm{s} \cdot \mathrm{cm}^{-5}\right)$ & $\chi^{ \pm s d}$ & $359.1 \pm 97.3$ & $232.2 \pm 22.75$ & .001 \\
TPG $(\mathrm{mmHg})$ & $\chi^{ \pm s d}$ & $11.95 \pm 5.08$ & $5.16 \pm 1.97$ & .001 \\
$\mathrm{CO}(\mathrm{l} / \mathrm{min})$ & $\chi^{ \pm s d}$ & $3.84 \pm 0.6$ & $4.11 \pm 0.5$ & .714 \\
$\mathrm{Cl}\left(\mathrm{l} / \mathrm{min} / \mathrm{m}^{2}\right)$ & $\chi^{ \pm s d}$ & $1.9 \pm 0.3$ & $2.2 \pm 0.4$
\end{tabular}

[Rivinius 2020]. Our trial confirmed these results and identified PVRI and TPG as independent predictors of postoperative outcome. PVRI had a hazard ratio of 1.006 (95\% [CI]: 1.001-1.012; $P=.018)$, while TPG had a hazard ratio of 1.172 (95\% [CI]: 1.032-1.033; $P=.015$ ).

The nature and specificity of the heart transplantation and terminal heart failure present an obstacle for randomization in research in this field, thus this represented a study limitation for our trial.

\section{CONCLUSION}

Identification of patients in terminal heart failure and pulmonary artery hypertension who are at higher risk for worse outcomes after HTx lends the opportunity to determine a treatment strategy and avoid postoperative complications. Pulmonary artery hypertension is an independent risk factor for higher 30-day mortality after HTx, while it did not affect the long-term outcome. Hemodynamic parameters obtained by right heart catheterization in heart transplant candidates could predict postoperative outcome. PVRI and TPG have been identified as independent predictors of higher 30-day postoperative mortality.

\section{REFERENCES}

Bourge RC, Kirklin JK, Naftel DC, White C, Mason DA, Epstein AE. 1991. Analysis and predictors of pulmonary vascular resistance after cardiac transplantation. J Thorac Cardiovasc Surg. 101(3): 432-444; discussion $444-435$

Butler J, Stankewicz MA, Wu J, et al. 2005. Pre-transplant reversible pulmonary hypertension predicts higher risk for mortality after cardiac transplantation. J Heart Lung Transplant. 24(2): 170-177.

Chang PP, Longenecker JC, Wang NY, et al. 2005. Mild vs severe pulmonary hypertension before heart transplantation: different effects on posttransplantation pulmonary hypertension and mortality. J Heart Lung Transplant. 24(8): 998-1007.

Costanzo MR, Dipchand A, Starling R, et al. 2010. The International Society of Heart and Lung Transplantation Guidelines for the care of heart transplant recipients. J Heart Lung Transplant. 29(8): 914-956.

D'Alonzo GE, Barst RJ, Ayres SM, et al. 1991. Survival in patients with primary pulmonary hypertension. Results from a national prospective registry. Ann Intern Med 115(5): 343-349.

Delgado JF, Gomez-Sanchez MA, Saenz de la Calzada C, et al. 2001. Impact of mild pulmonary hypertension on mortality and pulmonary artery pressure profile after heart transplantation. J Heart Lung Transplant. 20(9): 942-948.

Erickson KW, Costanzo-Nordin MR, O'Sullivan EJ, et al. 1990. Influence of preoperative transpulmonary gradient on late mortality after orthotopic heart transplantation. J Heart Transplant. 9(5): 526-537.

Hosenpud JD, Bennett LE, Keck BM, Boucek MM, Novick RJ. 2000. The Registry of the International Society for Heart and Lung Transplantation: seventeenth official report-2000. J Heart Lung Transplant. 19(10): 909-931.

Klotz S, Wenzelburger F, Stypmann J, Welp H, Drees G, Schmid C, Scheld HH. 2006. Reversible pulmonary hypertension in heart transplant candidates: to transplant or not to transplant. Ann Thorac Surg. 82(5): 1770-1773.

Lindelow B, Andersson B, Waagstein F, Bergh CH. 1999. High and low pulmonary vascular resistance in heart transplant candidates. A 5-year follow-up after heart transplantation shows continuous reduction in resistance and no difference in complication rate. Eur Heart J. 20(2): 148-156.

Mehra MR, Canter CE, Hannan MM, et al. 2016. International Society for Heart Lung Transplantation Infectious Diseases, F. Heart and C. Transplantation. The 2016 International Society for Heart Lung Transplantation listing criteria for heart transplantation: A 10-year update. J Heart Lung Transplant. 35(1): 1-23.

Mehra MR, Kobashigawa J, Starling R, et al. 2006. Listing criteria for heart transplantation: International Society for Heart and Lung Transplantation guidelines for the care of cardiac transplant candidates--2006. J Heart Lung Transplant. 25(9): 1024-1042.

Moreira N, Baptista R, Costa S, Franco F, Pego M, Antunes M. 2015. Lowering pulmonary wedge pressure after heart transplant: pulmonary compliance and resistance effect. Arq Bras Cardiol. 105(3): 292-300.

Murali S, Kormos RL, Uretsky BF, et al. 1993. Preoperative pulmonary hemodynamics and early mortality after orthotopic cardiac transplantation: the Pittsburgh experience. Am Heart J. 126(4): 896-904.

Rabin J and Kaczorowski DJ. 2019. Perioperative Management of the Cardiac Transplant Recipient. Crit Care Clin. 35(1): 45-60.

Rivinius R, Helmschrott M, Ruhparwar A, et al. 2020. Elevated pretransplant pulmonary vascular resistance is associated with early posttransplant atrial fibrillation and mortality. ESC Heart Fail. 7(1): 176-187.

Tenderich G, Koerner MM, Stuettgen B, et al. 1998. Does preexisting 
elevated pulmonary vascular resistance (transpulmonary gradient $>15$ $\mathrm{mm} \mathrm{Hg}$ or $>5$ wood) predict early and long-term results after orthotopic heart transplantation? Transplant Proc. 30(4): 1130-1131.

Vakil K, Duval S, Sharma A, et al. 2014. Impact of pre-transplant pulmonary hypertension on survival after heart transplantation: a UNOS registry analysis. Int J Cardiol. 176(3): 595-599.

Vassileva A, Valsecchi O, Sebastiani R, Fontana A, Gamba A. 2013. Heterotopic heart transplantation for elevated pulmonary vascular resistance in the current era: long-term clinical and hemodynamic outcomes. J Heart Lung Transplant. 32(9): 934-936. 